\title{
Decreased Platelet Counts and Serum Levels of VEGF-A, PDGF-BB, and BDNF in Extremely Preterm Infants Developing Severe ROP
}

\author{
Gunnel Hellgren ${ }^{a, b}$ Pia Lundgren ${ }^{b, c}$ Aldina Pivodic ${ }^{b} \quad$ Chatarina Löfqvist $^{b, d}$ \\ Anders K. Nilsson $^{b}$ David Ley ${ }^{\mathrm{e}}$ Karin Sävman ${ }^{\mathrm{f} g}$ Lois E. Smith ${ }^{\mathrm{h}}$ Ann Hellström ${ }^{b}$ \\ anstitute of Biomedicine, Sahlgrenska Academy, University of Gothenburg, Gothenburg, Sweden; ${ }^{\text {T} T h e ~ S a h l g r e n s k a ~}$ \\ Centre for Pediatric Ophthalmology Research, Department of Clinical Neuroscience, Institute of Neuroscience and \\ Physiology, Sahlgrenska Academy, University of Gothenburg, Gothenburg, Sweden; 'School of Medical Sciences, \\ Faculty of Medicine and Health, Örebro University, Örebro, Sweden; dInstitute of Health and Care Sciences, Sahlgrenska \\ Academy, University of Gothenburg, Gothenburg, Sweden; 'Department of Clinical Sciences Lund, Paediatrics, \\ Lund University, Skåne University Hospital, Lund, Sweden; 'Department of Pediatrics, Institute of Clinical Sciences, \\ Sahlgrenska Academy, University of Gothenburg, Gothenburg, Sweden; ${ }^{9}$ Region Västra Götaland, Department \\ of Neonatology, The Queen Silvia Children's Hospital, Sahlgrenska University Hospital, Gothenburg, Sweden; \\ hDepartment of Ophthalmology, Boston Children's Hospital, Harvard Medical School, Boston, MA, USA
}

\section{Keywords}

Retinopathy of prematurity · Platelets · Vascular endothelial growth factor - Platelet-derived growth factor .

Brain-derived neurotrophic factor

\begin{abstract}
Introduction: Thrombocytopenia has been identified as an independent risk factor for retinopathy of prematurity (ROP), although underlying mechanisms are unknown. In this study, the association of platelet count and serum plateletderived factors with ROP was investigated. Methods: Data for 78 infants born at gestational age $(\mathrm{GA})<28$ weeks were included. Infants were classified as having no/mild ROP or severe ROP. Serum levels of vascular endothelial growth factor $\mathrm{A}$, platelet-derived growth factor $\mathrm{BB}$, and brain-derived neurotrophic factor were measured in serum samples collected from birth until postmenstrual age (PMA) 40 weeks. Platelet counts were obtained from samples taken for clinical indication. Results: Postnatal platelet counts and serum concentrations of the 3 growth factors followed the same
\end{abstract}

karger@karger.com www.karger.com/neo

Karger $\stackrel{\text { ' }}{5}$

GOPEN ACCESS
(C) 2021 The Author(s)

Published by S. Karger AG, Basel

This is an Open Access article licensed under the Creative Commons Attribution-NonCommercial-4.0 International License (CC BY-NC) (http://www.karger.com/Services/OpenAccessLicense), applicable to the online version of the article only. Usage and distribution for commercial purposes requires written permission. postnatal pattern, with lower levels in infants developing severe ROP at PMA 32 and 36 weeks $(p<0.05-0.001)$. With adjustment for GA, low platelet counts and low serum concentrations of all 3 factors at PMA 32 weeks were significantly associated with severe ROP. Serum concentrations of all 3 factors also strongly correlated with platelet count $(p<$ 0.001). Conclusion: In this article, we show that ROP, platelet counts, and specific pro-angiogenic factors correlate. These data suggest that platelet-released factors might be involved in the regulation of retinal and systemic angiogenesis after extremely preterm birth. Further investigations are needed.

(c) 2021 The Author(s)

Published by S. Karger AG, Basel

\section{Introduction}

Infants born extremely preterm are immature and at high risk of developing both acute and long-lasting morbidities, including retinopathy of prematurity (ROP). In extremely preterm new-born infants, the retina is not ful- 
ly vascularized. At birth, a rapid change from the in utero milieu to the relatively hyperoxic ex utero milieu takes place. In the hyperoxic postnatal environment, local expression of oxygen-regulated pro-angiogenic factors decreases, and blood vessel development is suppressed in the first phase of ROP. With maturation of the neural retina, oxygen and nutrition demands increase, followed by enhanced expression of pro-angiogenic factors, including vascular endothelial growth factor A (VEGF-A). If ROP develops, this period constitutes the second phase of the disease, starting at approximately postmenstrual age (PMA) 30 weeks [1]. In this critical transition period, physiological vessel development or development of ROP occurs without or with pathological neovascularization. The exact mechanisms regulating retinal vascularization are not known.

Severe ROP is characterized by pathological retinal neovascularization that is visible several weeks to months after birth. Treatment usually begins at around 12 weeks postnatal age (PNA) or 36 weeks PMA. Immaturity, low birth weight, oxygen supplementation, and fluctuating oxygenation are well-established risk factors for the development of ROP, but several other risk factors have been described (reviewed in [2]). In recent years, thrombocytopenia has been identified as an independent risk factor for severe ROP [3-9], but the associated mechanisms are not understood. Proteomic studies have identified release of several pro-angiogenic, anti-angiogenic, and neurogenic factors in response to platelet activation, including VEGF, platelet-derived growth factor (PDGF), and brain-derived neurotrophic factor (BDNF) [10]. Some studies have suggested that ligand binding to endothelial cells followed by secretion of a-granule contents activates different signaling pathways involved in blood vessel growth and maturation. With this process, platelets might deposit angiogenic and neurogenic regulatory proteins in a localized manner $[11,12]$, but data are inconclusive [10]. Experimentally, factors released from activated platelets promote cell migration and endothelial cell tube formation $[13,14]$.

Regulation of vessel formation involves a complex interplay among different cell types. VEGF is a key player in this process. In rodents, VEGF protects from hyperoxia-induced vessel loss, and intraocular administration of anti-VEGF drugs suppresses hypoxia-induced neovascularization [15]. In the presence of a VEGF receptor antagonist, a compensatory angiogenic effect of platelet releasate has been observed in vitro [16].

A complex cross-talk among different cell types also regulates vascular maturation, involving endothelial, smooth muscle, retinal ganglion, and Müller cells, along with astrocytes and pericytes. Platelet-derived growth factor BB (PDGF-BB) has been described as a connecting factor between endothelial cells and retinal ganglion cells, astrocytes, and Müller cells during angiogenesis, and as a key factor for pericyte recruitment [17]. In mice, administration of PDGF-BB affects retinal vascular remodeling in a time-dependent manner through different developmental stages [18]. Only pericytes engaged in ongoing cell migration respond to exogenous PDGF-BB, suggesting a shift in responsiveness upon maturation. In mice, antagonism of both PDGF-BB and VEGF-A has an additive suppressive effect on pathological neovascularization. The period of VEGF dependence corresponds to the period before new vessels acquire a pericyte coating $[18,19]$.

The expression of BDNF in ganglion cells, astrocytes, and Müller cells was described in the 1990s. More recently, BDNF expression has also been reported in endothelial cells [20]. We and others have found lower levels of circulating BDNF in blood samples from infants with ROP compared to infants without ROP [21-23]. In addition, although genetic variants in the BDNF gene have been associated with severe ROP $[24,25]$, data are inconclusive [26].

Among extremely preterm infants, thrombocytopenia is one of the most common hematologic conditions in the neonatal period. Its prevalence is inversely correlated with gestational age (GA) at birth [27]. We speculate that thrombocytopenia is a risk factor for ROP, associated at least partly with decreased levels of locally released platelet-derived angiogenic and neurotrophic factors because of a low platelet count. Most platelet-derived factors are expressed in a diversity of cells, and the origin of measured concentrations in the circulation is diverse. However, levels of serum VEGF-A and BDNF are highly correlated with platelet count, supporting the hypothesis that these values in serum originate to a high degree from released $\alpha$-granule content [28-30]. Furthermore, serum concentrations of VEGF increase with clotting time [31], and BDNF concentrations are low in platelet-poor plasma, whereas concentrations are approximately 100-200fold higher in pooled serum than in plasma because of release during the in vitro clotting process. Given this pattern, serum levels could be used as an indirect measurement of platelet-derived levels of these factors. The aim of this study was to investigate the associations between plasma platelet count and serum levels of selected platelet-derived factors with severe ROP in a cohort of extremely preterm infants. 
Table 1. Clinical characteristics

\begin{tabular}{lcccc}
\hline & $\begin{array}{l}\text { No ROP } \\
n=17\end{array}$ & $\begin{array}{l}\text { Mild ROP } \\
\text { (stage 1 and 2) } \\
n=30\end{array}$ & $\begin{array}{l}\text { Severe ROP } \\
\text { (stage 3 or treated) } \\
n=31\end{array}$ & $\begin{array}{l}\text { All } \\
N=78\end{array}$ \\
\hline GA at birth, mean (SD), wk & $26.1(1.4)$ & $25.4(1.3)$ & $24.3(1.1)$ & $25.1(1.4)$ \\
Birth weight, mean (SD), g & $1,024(194)$ & $776(199)$ & $693(170)$ & $797(223)$ \\
Weight SD score, mean (SD) & $-0.10(0.87)$ & $-1.20(1.27)$ & $-0.85(1.24)$ & $-0.82(1.20)$ \\
Male, $n(\%)$ & $13(76)$ & $11(37)$ & $19(61)$ & $43(55)$ \\
Bronchopulmonary dysplasia, $n(\%)$ & $4(24)$ & $15(50)$ & $20(65)$ & $39(50)$ \\
Necrotizing enterocolitis & $0(0)$ & $1(3.3)$ & $4(13)$ & $5(6.4)$ \\
Patent ductus arteriosus & $6(35)$ & $29(97)$ & $26(81)$ & $54(69)$ \\
Sepsis, $n$ (\%) & $3(18)$ & $12(40)$ & $15(48)$ & $30(39)$ \\
Thrombocytopenia, $<100 \times 10^{9} / \mathrm{L}, n(\%)$ & $2(12)$ & $13(43)$ & $23(74)$ & $38(49)$ \\
Platelet transfusion & $0(0)$ & $6(20)$ & $18(58)$ & $24(31)$ \\
\hline
\end{tabular}

GA, gestational age; ROP, retinopathy of prematurity; SD, standard deviation.

\section{Methods}

\section{Study Design}

The study was performed within the Donna Mega Study, a randomized, open-label, controlled trial conducted at Queen Silvia Children's Hospital in Gothenburg, Sweden (NCT 02760472). Briefly, infants born at $<28$ weeks GA were randomly allocated to parenteral lipid emulsion with SMOFlipid ${ }^{\circledR}$ or with Clinoleic ${ }^{\circledR}$. Exclusion criteria were major congenital malformations. The primary outcome was ROP, as described by Najm et al. [32]. Secondary analyses included evaluation of postnatal angiogenic and neurotrophic biomarkers in relation to ROP. The study design has been described previously [32].

\section{Study Population}

From April 2013 to September 2015, parents of 90 of 138 eligible infants agreed to participate in the study. Of these, data for 78 infants (43 male; 55\%, mean [SD] GA, 25.5 [1.4] weeks) with a known ROP outcome were evaluated in the final analyses. Laser therapy was used for all infants treated for ROP in this study.

\section{Eye Examinations}

ROP screening started at PNA 5-6 weeks but not before PMA 31 weeks. Retinal examinations through dilated pupils were performed at a rate of twice weekly to every 2 weeks, depending on ROP severity, until the retina was fully vascularized or the condition was considered stable. ROP was classified based on the international classification [33], and the recommendations of the Early Treatment for Retinopathy of Prematurity Cooperative Group [34] were followed for treatment. The study outcome of severe ROP was defined as stage 3 and/or treated ROP.

\section{Morbidities}

Diagnoses, including bronchopulmonary dysplasia, necrotizing enterocolitis, persistent arteriosus, sepsis, and thrombocytopenia, were retrieved from clinical records. Bronchopulmonary dysplasia was defined as moderate-to-severe lung disease with a need for oxygen supplementation at PMA 36 weeks, necrotizing enterocolitis was diagnosed by clinical signs and radiologic findings
(Bell's stages 2-3), and persistent arteriosus was registered when the infant had clinical symptoms that required either pharmacological or surgical treatment. Sepsis was diagnosed by clinical symptoms accompanied by a positive blood culture. When the culture contained Staphylococcus epidermidis, an elevated C-reactive protein $(>20 \mathrm{mg} / \mathrm{L})$ was required for diagnosis. National recommendations defined neonatal thrombocytopenia as a platelet count $<100 \times 10^{9} / \mathrm{L}$.

\section{Blood Sampling and Laboratory Analyses}

Serum samples were collected at birth (cord blood); at postnatal days 1, 7, 14, and 28; and at PMA 32, 36, and 40 weeks. Samples were centrifuged, aliquoted, and placed at $-70^{\circ} \mathrm{C}$ within $2 \mathrm{~h}$ of being taken. VEGF-A, BDNF, and PDGF-BB were analyzed using the ELLA multi-analyte platform (Biotechne, Minneapolis, MN, USA) according to the protocol provided by the manufacturer. Briefly, $50 \mu \mathrm{L}$ sample, diluted 1:4 in SD13 buffer, was placed in cartridge wells, and then the atomized analysis procedure was finalized within 70 min. Raw data were processed using Simple Plex Explorer Software (Biotechne). Inter-assay coefficients of variation (CVs) tested among 40 assays were $7.00,7.09$, and $7.76 \%$ at 35,475 , and $1,667 \mathrm{pg} / \mathrm{mL}$, respectively, for VEGF-A; 7.05, 6.83, and $5.01 \%$ at $220,7,402$, and $10,271 \mathrm{pg} / \mathrm{mL}$, respectively, for BDNF; and 4.51, 3.60 , and $4.69 \%$ at $7.5,332$, and $945 \mathrm{pg} / \mathrm{mL}$, respectively, for PDGFBB. Intra-assay CVs based on 9 measurements of a pooled serum sample were $4.77 \%$ at $502 \mathrm{pg} / \mathrm{mL}$ for VEGF-A, $5.51 \%$ at $7,731 \mathrm{pg} /$ $\mathrm{mL}$ for BDNF, and $5.24 \%$ at $983 \mathrm{pg} / \mathrm{mL}$ for PDGF-BB.

Plasma samples for platelet counts were collected for clinical indications and analyzed by Advia 2120i (Siemens Healthcare $\mathrm{GmbH}$, Erlangen, Germany) and a modified method in Technicon H6000. The same methods were used at all hospitals in the Västra Götaland region where infants were hospitalized. For each infant, mean platelet count per day was performed and a mean weekly platelet count calculated.

\section{Statistics}

Mean and SD are presented for normally distributed continuous variables and median and range for skewed continuous variables. The Mann-Whitney U test was used for comparisons be- 


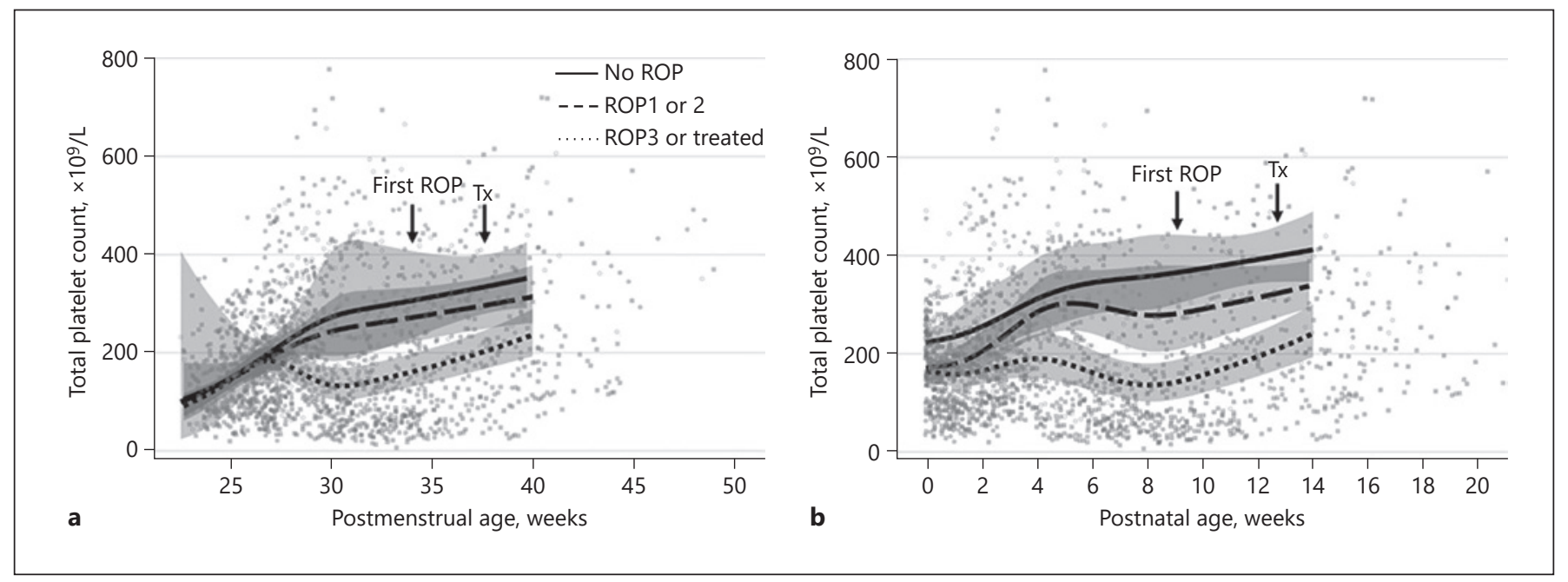

Fig. 1. Platelet counts, with means and 95\% CIs shown for infants without ROP or with mild ROP (stage 1 or 2 ) or severe ROP (stage 3, untreated and treated), respectively. Indicated with arrows are mean age when ROP was first detected and mean age for treatment (Tx). a Values corresponding to PMA. b Values corresponding to postnatal age. CI, confidence interval; ROP, retinopathy of prematurity; PMA, postmenstrual age.

tween 2 independent groups. For descriptive purposes, random coefficient models were applied when estimating mean and 95\% confidence interval (CI) for platelet count, VEGF-A, BDNF, and PDGF-BB for no, mild, and severe ROP handling of the underlying timescales, PMA and PNA in separate analyses, using natural cubic splines. Residual plots were reviewed and robust sandwich estimators used to adjust for heteroscedasticity. Univariable and GA-adjusted logistic regression analyses were performed for evaluation of the association of VEGF-A, BDNF, and PDGF-BB with severe ROP. ORs with $95 \%$ CIs, and c-statistics with 5\% CIs are presented. A model with a c-statistic $>0.70$ was considered acceptable. Missing data were not imputed.

Relationships between platelet count and VEGF-A, BDNF, or PDGF-BB were investigated using Spearman correlations. All statistical analyses were performed using SPSS 23 for Microsoft Windows (IBM, Armonk, NY, USA) and SAS Software version 9.4 (SAS Institute Inc., Cary, NC, USA), with two-tailed tests and a $p$ value $<0.05$ considered as significant.

\section{Results}

\section{Clinical Characteristics}

The clinical characteristics of this study population have been described previously $[32,35]$. Table 1 shows the clinical characteristics for this cohort, stratified into "no ROP" ( $n=17)$, "mild ROP" (stage 1 or $2 ; n=30$ ), and "severe ROP" (stage 3 or 4 , untreated or treated; $n=31$ ).

Lower Platelet Count in Infants with Proliferative ROP

Platelet count in relation to ROP was investigated by weekly mean platelet count in samples taken for clinical indications, according to both PMA and PNA. As shown in Figure 1, the numerical differences in platelet counts between infants without ROP and with mild ROP were quite small. However, infants with severe ROP had a lower platelet count from PMA 30 weeks (shown in Fig. 1a) and PNA 5 weeks (shown in Fig. 1b). For statistical analyses, infants were grouped as non/mild ROP (non-ROP, $n=17$; ROP stage $1, n=8$; ROP stage $2, n=22$ ) or severe ROP $(n=23)$. Mann-Whitney $\mathrm{U}$ results confirmed lower platelet counts in infants with severe ROP than in infants with no or mild ROP at PMA 32 and 36 weeks (shown in Table 2). Regarding PNA, sufficient data for statistical analyses were available only for 4 weeks after birth; however, the temporal pattern according to PNA followed the same pattern as for PMA.

Logistic regression was used for further analysis of the association between low platelet count and ROP. Platelet count was investigated per 50 units by univariate logistic regression analyses, and in a second step adjusted for GA at birth. In univariate analysis, low platelet count was identified as a predictor for severe ROP at PMA 32 (OR 0.70 [95\% CI 0.56-0.87] per 50-unit increase; $p=0.002$ ) and 36 weeks (OR 0.61 [95\% CI 0.43-0.87] per 50-unit increase; $p=0.021$ ). After adjustment for GA, however, only low platelet count at PMA 36 weeks remained significant (OR 0.65 [95\% CI 0.45-0.94]; $p=0.021$ ) among the 33 infants whose data were available (shown in Table 3 ). The c-statistics for the univariable model with total platelet count at PMA 36 weeks PMA was high (0.82; 95\% CI 0.66-0.98). 


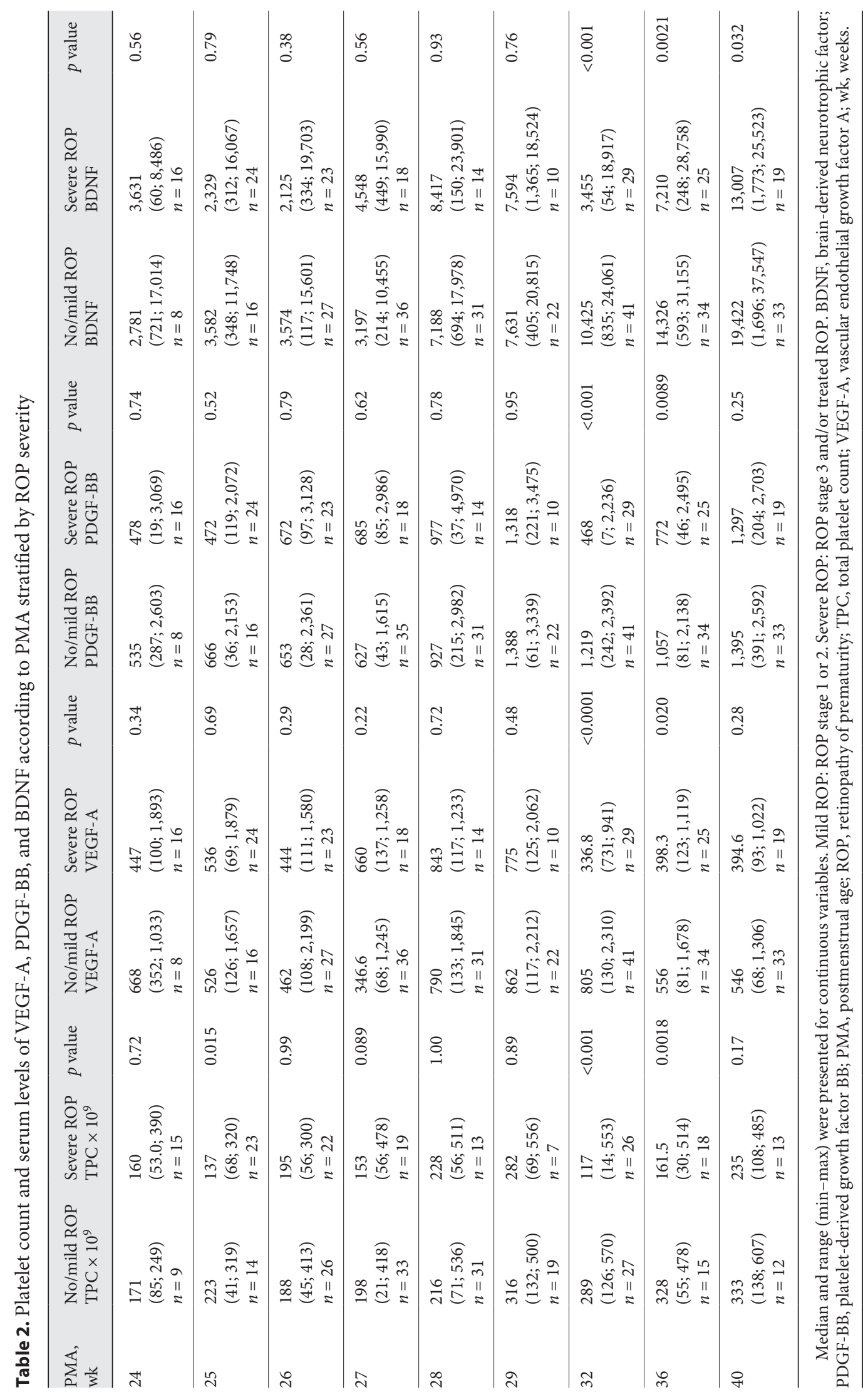




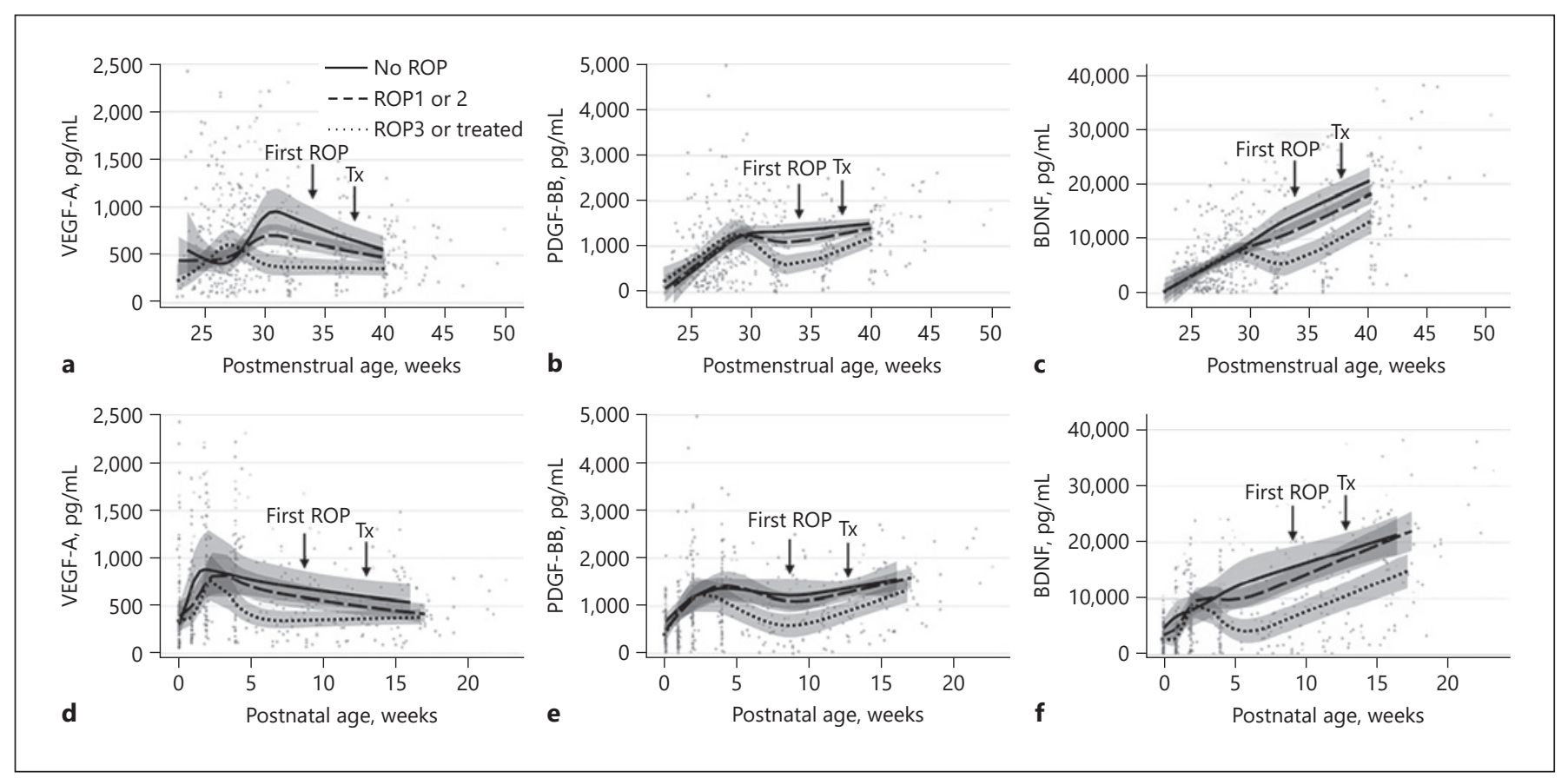

Fig. 2. Serum concentrations of VEGF-A (a, d), PDGF-BB (b, e), and BDNF (c, f), with means and $95 \%$ CIs shown for infants with no ROP or with mild ROP (stage 1 or 2) or severe ROP (stage 3, untreated and treated), respectively. Arrows indicate mean age when ROP was first detected and mean age for treatment (Tx). Upper panel shows values corresponding to PMA and lower panel shows values corresponding to postnatal age. CI, confidence interval; ROP, retinopathy of prematurity; VEGF-A, vascular endothelial growth factor A; PDGF-BB, plateletderived growth factor BB; BDNF, brain-derived neurotrophic factor; PMA, postmenstrual age.

\section{BDNF, VEGF-A, and PDGF-BB Associated with}

Severe ROP in the Second Phase of ROP

The longitudinal pattern for VEGF-A, PDGF-BB, and BDNF in infants with no, mild, or severe ROP was similar to the pattern shown for platelet count, with lower levels from PMA approximately 30 weeks (shown in Fig. 2a-c) or PNA approximately 5 weeks (shown in Fig. $2 \mathrm{~d}-\mathrm{f}$ ) in infants developing severe ROP compared to infants with no or mild ROP. According to PMA, descriptive statistics revealed lower VEGF-A, PDGF-BB, and BDNF levels in infants developing severe ROP compared to those with no or mild ROP (shown in Table 2). By PNA, enough data for statistical analyses were available only to PNA 4 weeks. During the first weeks of life, serum concentrations of PDGF-BB and VEGF-A were similar regardless of later ROP. However, BDNF levels were lower at PNA 1 and 4 weeks in infants developing severe ROP compared to levels in infants with no or mild ROP. Median BDNF (min; $\max )$ at PNA 1 week were $2,035(60 ; 15,232) \mathrm{pg} / \mathrm{mL}$ in infants developing severe ROP, compared to 4,264 (257; $17,978) \mathrm{pg} / \mathrm{mL}$ in infants with no or mild ROP $(p=0.038)$. At PNA 4 weeks, corresponding levels of BDNF were
$5,122(270 ; 18,524) \mathrm{pg} / \mathrm{mL}$ in infants developing severe ROP compared to $9,626(405 ; 24,154) \mathrm{pg} / \mathrm{mL}$ in infants with no or mild ROP $(p=0.003)$.

According to PMA, univariable logistic regression analyses identified low levels of VEGF-A, PDGF-BB, and BDNF at PMA 32 and 36 weeks and BDNF at PMA 40 weeks as associated with severe ROP. After adjustment for GA, low serum levels of VEGF-A, PDGF-BB, and BDNF at PMA 32 weeks remained significant. All 3 variables at PMA 32 weeks showed high predictive ability when analyzed alone, as well, with c-statistics from 0.80 to 0.85 (details shown in Table 3 ).

\section{Correlation of BDNF, VEGF, and PDGF with Platelet Count}

We used correlation analyses to investigate the relationship between platelet count and serum levels of VEGF-A, PDGF-BB, and BDNF. Only platelet values obtained from samples taken the same day as VEGF-A, PDGF-BB, and BDNF were included. Results showed a moderate to strong correlation between platelet count and VEGF-A, PDGF-BB, and BDNF, respectively $(r=$ 
Table 3. Analysis of VEGF-A, PDGF-BB, and BDNF serum levels as risk factors for severe ROP using logistic regression, before and after adjustment for GA at birth

\begin{tabular}{|c|c|c|c|c|c|}
\hline \multirow[t]{2}{*}{ Variables } & \multicolumn{3}{|l|}{ Univariable } & \multicolumn{2}{|l|}{ Adjusted for GA } \\
\hline & $\begin{array}{l}\text { OR }(95 \% \mathrm{CI}) \\
\text { severe ROP }\end{array}$ & $p$ value & $\begin{array}{l}\text { c-statistics } \\
(95 \% \mathrm{CI})\end{array}$ & $\begin{array}{l}\text { OR }(95 \% \mathrm{CI}) \\
\text { severe ROP }\end{array}$ & $p$ value \\
\hline TPC at PMA 32 weeks (OR per $\left.50 \times 10^{9} / \mathrm{L}\right) n=53$ & $0.70(0.56-0.87)$ & 0.002 & $0.82(0.69-0.94)$ & $0.79(0.62-1.02)$ & 0.067 \\
\hline TPC at PMA 36 weeks (OR per $\left.50 \times 10^{9} / \mathrm{L}\right) n=33$ & $0.61(0.43-0.87)$ & 0.006 & $0.82(0.66-0.98)$ & $0.65(0.45-0.94)$ & 0.021 \\
\hline VEGF-A at PMA 32 weeks (OR per $100 \mathrm{pg} / \mathrm{mL}$ ), $n=70$ & $0.64(0.51-0.80)$ & $<0.001$ & $0.85(0.76-0.94)$ & $0.68(0.54-0.85)$ & $<0.001$ \\
\hline VEGF-A at PMA 36 weeks (OR per $100 \mathrm{pg} / \mathrm{mL}$ ), $n=59$ & $0.81(0.67-0.98)$ & 0.029 & $0.68(0.54-0.82)$ & $0.87(0.70-1.06)$ & 0.17 \\
\hline VEGF-A at PMA 40 weeks (OR per $100 \mathrm{pg} / \mathrm{mL}$ ), $n=52$ & $0.87(0.70-1.08)$ & 0.21 & $0.59(0.43-0.76)$ & $0.88(0.68-1.14)$ & 0.33 \\
\hline PDGF-BB at PMA 32 weeks (OR per $100 \mathrm{pg} / \mathrm{mL}$ ), $n=70$ & $0.79(0.70-0.89)$ & $<0.001$ & $0.83(0.72-0.94)$ & $0.84(0.75-0.95)$ & 0.005 \\
\hline PDGF-BB at PMA 36 weeks (OR per $100 \mathrm{pg} / \mathrm{mL}$ ), $n=59$ & $0.89(0.79-0.99)$ & 0.030 & $0.70(0.56-0.85)$ & $0.92(0.82-1.03)$ & 0.16 \\
\hline PDGF-BB at PMA 40 weeks (OR per $100 \mathrm{pg} / \mathrm{mL}$ ), $n=52$ & $0.94(0.84-1.04)$ & 0.22 & $0.60(0.42-0.77)$ & $0.94(0.83-1.06)$ & 0.29 \\
\hline BDNF at PMA 32 weeks (OR per 1,000 pg/mL), $n=70$ & $0.82(0.73-0.92)$ & $<0.001$ & $0.80(0.69-0.91)$ & $0.87(0.78-0.98)$ & 0.021 \\
\hline BDNF at PMA 36 weeks (OR per $1,000 \mathrm{pg} / \mathrm{mL}$ ), $n=59$ & $0.89(0.82-0.97)$ & 0.005 & $0.74(0.60-0.87)$ & $0.93(0.85-1.01)$ & 0.077 \\
\hline BDNF at PMA 40 weeks (OR per $1,000 \mathrm{pg} / \mathrm{mL}$ ), $n=52$ & $0.91(0.84-0.99)$ & 0.025 & $0.68(0.53-0.84)$ & $0.92(0.84-1.01)$ & 0.089 \\
\hline
\end{tabular}

Severe ROP: ROP stage 3 and/or treated ROP. BDNF, brain-derived neurotrophic factor; GA, gestational age; OR, odds ratio; CI, confidence interval; PDGF-BB, platelet-derived growth factor BB; PMA, postmenstrual age; ROP, retinopathy of prematurity; TPC, total platelet count; VEGF-A, vascular endothelial growth factor A; wk, weeks.

$0.42, r=0.66$ and $r=0.70$, respectively; $p<0.001$ ) (shown in Fig. 3). In addition, pairwise correlation analyses for VEGF-A, BDNF, and PDGF-BB revealed moderate-tostrong correlations among the factors (VEGF-A vs. PDGF-BB, $r_{\mathrm{s}}=0.65$; VEGF-A vs. BDNF, $r_{\mathrm{s}}=0.52$; and BDNF vs. PDGF-BB, $\left.r_{\mathrm{s}}=0.78 ; p<0.001\right)$.

\section{Discussion}

The current results represent the first findings of a strong correlation between low platelet count and low serum levels of VEGF-A, PDGF-BB, and BDNF, from PMA of approximately 30 weeks and PNA of 5 weeks in infants developing severe ROP. Although platelets are carriers of a number of pro- and anti-angiogenic factors, and low platelet count or thrombocytopenia is an independent risk factor for ROP $[3,4,7,9,36,37]$, the specific factors behind the association have been unclear.

From around PMA 30 weeks, during the second phase of ROP, the peripheral retina becomes hypoxic. VEGF is upregulated, and some infants develop proliferative ROP, which is treated with the aim of reducing retinal VEGF. It was during this phase that we found an association of low platelet counts and low levels of circulating growth factors with severe ROP. These results are not associated with external supply of platelets by transfusion. Infants developing severe ROP receive platelet transfusion most frequently. In addition, most platelet transfusions are giv- en earlier than the PMA 30 weeks. Platelets are carriers of both pro- and anti-angiogenic factors and might function to prevent retinal neovascularization. In a mouse model of ROP, platelet depletion increased neovascularization, whereas platelet transfusion at postnatal day $(\mathrm{P}) 15$ and P16 suppressed neovascularization. These effects were mediated by factors released from platelet granules [36]. In these animals, retinal VEGF mRNA and protein expression increased upon platelet depletion and decreased after transfusion, supporting the hypothesis of a local anti-angiogenic effect of platelets.

In the late 1960s and early 1970s, Gimbron et al. [40] and others reported that platelets not only regulate hemostasis but also influence new blood vessel development and that thrombocytopenia leads to elevated vascular permeability [38-40]. Furthermore, platelet activity changes through developmental stages. Neonatal platelets have been characterized as hyporeactive compared to adult platelets and also compared to platelets from fullterm infants in some studies (reviewed in [41]).

Platelet $\alpha$-granules contain high amounts of both proand anti-angiogenic factors, but it is generally accepted that activation of platelets has an overall stimulatory net effect on angiogenesis. In vitro, releasate from activated platelets induces tube formation and cell migration of human umbilical cord endothelial vein cells [14]. Heat treatment negatively affects cell proliferation, suggesting that at least some activation factors are proteins. 


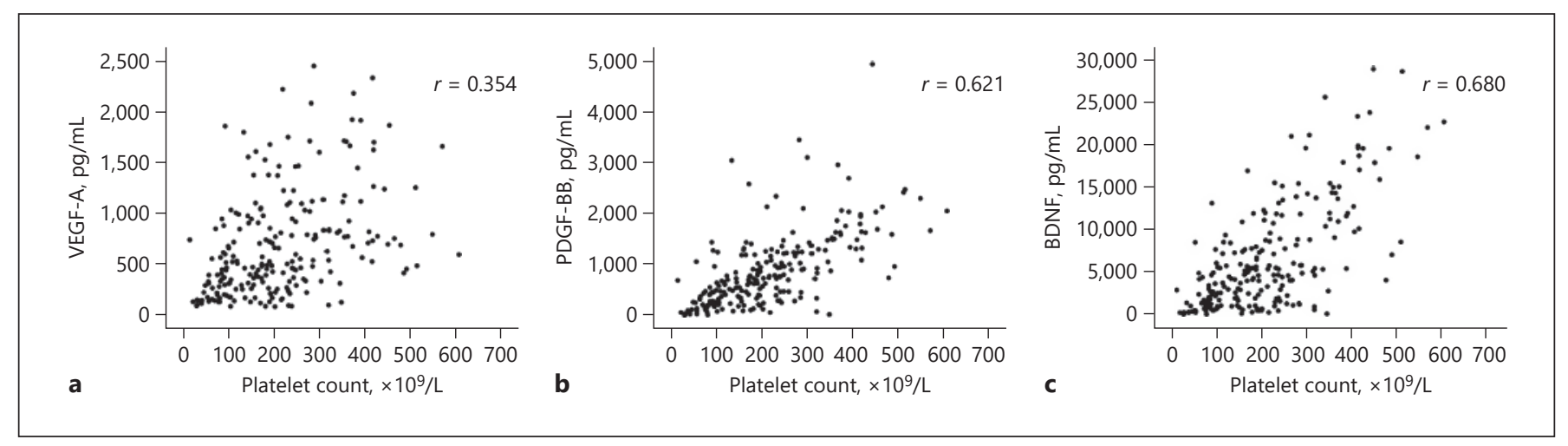

Fig. 3. Correlation of platelet counts with VEGF-A (a), PDGF-BB (b), and BDNF (c). VEGF-A, vascular endothelial growth factor A; PDGF-BB, platelet-derived growth factor BB; BDNF, brain-derived neurotrophic factor.

After preterm birth, physiological retinal vascularization probably depends on timing and the availability of pro- and anti-angiogenic factors. This idea is supported by findings in neonates with and without ROP and in rats of different ratios of pro-angiogenic VEGF/anti-angiogenic pigment epithelial-derived factor $[42,43]$.

In this study, the temporal patterns of the pro-angiogenic factors VEGF-A and PDGF-BB, as well as the neurotrophic factor BDNF, were evaluated in relation to platelet count and to ROP. It has previously been shown that high levels of these factors are stored in platelet a-granules and released as a response to activation. Levels measured in serum mainly originate from platelet release in response to in vitro coagulation [28, 31, 44, 45]. These previous findings together with the strong correlation identified in the present study of VEGF-A, PDGF-BB, and $\mathrm{BDNF}$ in serum with platelet counts in plasma suggest that serum levels of at least these factors mainly are carried by platelets and released during clotting. The role of VEGF-A in the development of ROP is well established, and PDGF-BB and BDNF also have been associated with ROP [18, 19, 21-24, 46, 47].

Data are inconclusive regarding systemic levels of VEGF in relation to ROP [48]. In the current work, VEGF-A levels during the first postnatal weeks were similar and independent of later ROP. From a PMA of approximately 30 weeks, infants developing severe ROP had lower VEGF-A than infants with no or mild ROP. These results contrast with our previous data showing increased circulating VEGF levels prior to the first signs of ROP [49] but are in line with other studies demonstrating the same postnatal pattern. The reasons for the contrasting results are not clear. However, in our previous study, weekly samples were collected from birth until PMA 40 weeks, whereas in the present study, samples were collected at birth, 1,2, and 4 weeks after birth, and then with a lower resolution at PMA 32, 36, and 40 weeks. Variations in the temporal pattern thus could have been missed. Furthermore, systemic VEGF levels are highly variable within and among individuals, and small sample numbers might have contributed to the divergent results between the studies. Today it is not known how, or if, these factors are influenced by ROP treatment. This could be further elucidated in a follow-up study where samples are collected with higher resolution around the time when proliferative ROP is detected and around the time for treatment.

Our findings do not offer mechanistic explanations for what underlies thrombocytopenia as a risk factor for ROP. This is a limitation and requires investigation. Of interest, in vitro, selective $\alpha$-granule release depending on stimulatory agents has been reported. For example, thromboxane $\mathrm{A} 2$ and adenosine diphosphate stimulation result in opposite effects on VEGF release, cell migration, and tube formation [50]. In vivo, selective release of proor anti-angiogenic factors might be involved in the regulation of the local availability of these factors, and in this way, the regulation of vessel development.

In summary, results from this study show a strong correlation of the postnatal pattern of platelet count with serum levels of the neurogenic and angiogenic factors VEGF-A, PDGF-BB, and BDNF. Low platelet count during the second phase of ROP was associated with low levels of these factors. Although increased retinal levels of VEGF during the hypoxic second phase of ROP cause uncontrolled neovascularization that needs treatment to lower retinal VEGF concentrations, we found decreased serum VEGF concentrations during this same period. 
Taking our clinical findings together with previously published experimental findings, we suggest that depending on the physiological condition, platelet-released factor might be involved in the regulation of retinal angiogenesis after extremely preterm birth. Experimental studies are needed to further elucidate mechanisms underlying thrombocytopenia as a risk factor for ROP.

\section{Acknowledgements}

The authors thank all the participants and the study team led by Carola Pfeiffer-Mosesson, Anne Rosenqvist, Mary Gustafsson, Svetlana Najm, Clara Mannheimer, and Anna Thurfjell for their very valuable help with retrieving data for the study.

\section{Statement of Ethics}

The study was performed according to the World Medical Association Declaration of Helsinki, and the trial was approved by the Regional Ethical Board, Gothenburg (Dnr 303-11), at the University of Gothenburg. Informed written consent was obtained for all participants from their parents or guardians. No manufacturer of the parenteral products contributed to the design of the study, accrual or analysis of the data, or preparation of the manuscript.

\section{Conflict of Interest Statement}

The authors declare no conflicts of interest to declare.

\section{Funding Sources}

This study was supported by grants provided by the Swedish Medical Research Council \#2016-01131, The Gothenburg Medical Society, Government grants under ALF agreement ALFGBG-717971, Knut and Alice Wallenberg Foundation, De Blindas Vänner, Kronprinsessan Margaretas Arbetsnämnd för synskadade, Cronqvists foundation, Örebro County Council Research Committee, \#OLL-933343, and National Institutes of Health EY017017, EY024868, BCH IDDRC, and 1U54HD090255 P01 HD18655.

\section{Author Contributions}

G.H., P.L. and A.H. contributed to the conception of the research; all authors contributed to the design of the research; G.H., P.L., and A.P. contributed to the acquisition and analysis of the data; all authors contributed to the interpretation of the data; and G.H. drafted the manuscript. All authors critically revised the manuscript, and approved the final manuscript.

\section{References}

1 Smith LE. Pathogenesis of retinopathy of prematurity. Growth Horm IGF Res. 2004; 14(Suppl A):S140-4.

2 Kim SJ, Port AD, Swan R, Campbell JP, Chan RVP, Chiang MF. Retinopathy of prematurity: a review of risk factors and their clinical significance. Surv Ophthalmol. 2018 SepOct;63(5):618-37.

3 Jensen AK, Ying GS, Huang J, Karp K, Quinn GE, Binenbaum G. Thrombocytopenia and retinopathy of prematurity. J AAPOS. 2011; 15(1):e3-4.

4 Lundgren P, Lundberg L, Hellgren G, Holmström G, Hård AL, Smith LE, et al. Aggressive posterior retinopathy of prematurity is associated with multiple infectious episodes and thrombocytopenia. Neonatology. 2017 111(1):79-85.

5 Rastogi S, Olmez I, Bhutada A, Rastogi D. Drop in platelet counts in extremely preterm neonates and its association with clinical outcomes. J Pediatr Hematol Oncol. 2011;33(8) $580-4$.

6 Tao Y, Dong Y, Lu CW, Yang W, Li Q. Relationship between mean platelet volume and retinopathy of prematurity. Graefes Arch Clin Exp Ophthalmol. 2015;253(10):1791-4.

7 Vinekar A, Hegde K, Gilbert C, Braganza S, Pradeep M, Shetty R, et al. Do platelets have a role in the pathogenesis of aggressive poste- rior retinopathy of prematurity? Retina. 2010; 30(4 Suppl):S20-3.

8 Yau GS, Lee JW, Tam VT, Yip S, Cheng E, Liu CC, et al. Differences in risk factors for retinopathy of prematurity development in paired twins: a chinese population study. ScientificWorldJournal. 2014;2014:212183.

9 Jensen AK, Ying GS, Huang J, Quinn GE, Binenbaum G. Longitudinal study of the association between thrombocytopenia and retinopathy of prematurity. J AAPOS. 2018; 22(2):119-23

10 van Holten TC, Bleijerveld OB, Wijten P, de Groot PG, Heck AJ, Barendrecht AD, et al. Quantitative proteomics analysis reveals similar release profiles following specific PAR-1 or PAR-4 stimulation of platelets. Cardiovasc Res. 2014;103(1):140-6.

11 Italiano JE Jr, Richardson JL, Patel-Hett S, Battinelli E, Zaslavsky A, Short S, et al. Angiogenesis is regulated by a novel mechanism: proand antiangiogenic proteins are organized into separate platelet alpha granules and differentially released. Blood. 2008;111(3):1227-33.

12 Walsh TG, Metharom P, Berndt MC. The functional role of platelets in the regulation of angiogenesis. Platelets. 2015;26(3):199-211.

13 Brill A, Elinav H, Varon D. Differential role of platelet granular mediators in angiogenesis. Cardiovasc Res. 2004;63(2):226-35.
14 Kim HK, Song KS, Chung JH, Lee KR, Lee SN Platelet microparticles induce angiogenesis in vitro. Br J Haematol. 2004;124(3):376-84.

15 Ferrara N. Vascular endothelial growth factor: basic science and clinical progress. Endocr Rev. 2004;25(4):581-611.

16 Etulain J, Fondevila C, Negrotto S, Schattner $\mathrm{M}$. Platelet-mediated angiogenesis is independent of VEGF and fully inhibited by aspirin. Br J Pharmacol. 2013;170(2):255-65.

17 Lindahl P, Johansson BR, Levéen P, Betsholtz C. Pericyte loss and microaneurysm formation in PDGF-B-deficient mice. Science. 1997; 277(5323):242-5

18 Benjamin LE, Hemo I, Keshet E. A plasticity window for blood vessel remodelling is defined by pericyte coverage of the preformed endothelial network and is regulated by PDGF-B and VEGF. Development. 1998;125(9):1591-8.

19 Dong A, Seidel C, Snell D, Ekawardhani S, Ahlskog JK, Baumann M, et al. Antagonism of PDGF-BB suppresses subretinal neovascularization and enhances the effects of blocking VEGF-A. Angiogenesis. 2014;17(3):55362.

20 Nakahashi T, Fujimura H, Altar CA, Li J, Kambayashi J, Tandon NN, et al. Vascular endothelial cells synthesize and secrete brainderived neurotrophic factor. FEBS Lett. 2000; 470(2):113-7. 
21 Hellgren G, Willett K, Engstrom E, Thorsen P, Hougaard DM, Jacobsson B, et al. Proliferative retinopathy is associated with impaired increase in bdnf and rantes expression levels after preterm birth. Neonatology. 2010; 98(4):409-18.

22 Rao R, Mashburn CB, Mao J, Wadhwa N, Smith GM, Desai NS. Brain-derived neurotrophic factor in infants $<32$ weeks gestational age: correlation with antenatal factors and postnatal outcomes. Pediatr Res. 2009;65: 548-52.

23 Sood BG, Madan A, Saha S, Schendel D, Thorsen P, Skogstrand K, et al. Perinatal systemic inflammatory response syndrome and retinopathy of prematurity. Pediatr Res. 2010; 67(4):394-400.

24 Hartnett ME, Morrison MA, Smith S, Yanovitch TL, Young TL, Colaizy T, et al. Genetic variants associated with severe retinopathy of prematurity in extremely low birth weight infants. Invest Ophthalmol Vis Sci. 2014;55(10): 6194-203.

25 Hartnett ME, Cotten CM. Genomics in the neonatal nursery: focus on rop. Semin Perinatol. 2015;39(8):604-10.

26 Lasabova Z, Stanclova A, Grendar M, Mikolajcikova S, Calkovska A, Lenhartova N, et al. Genetic association of single nucleotide polymorphisms of FZD4 and bdnf genes with retinopathy of prematurity. Ophthalmic Genet. 2018;1-6.

27 Stanworth SJ. Thrombocytopenia, bleeding, and use of platelet transfusions in sick neonates. Hematol Am Soc Hematol Educ Program. 2012;2012:512-6.

28 Gunsilius E, Petzer A, Stockhammer G, Nussbaumer W, Schumacher P, Clausen J, et al. Thrombocytes are the major source for soluble vascular endothelial growth factor in peripheral blood. Oncology. 2000;58(2):169-74.

29 Naegelin Y, Dingsdale H, Sauberli K, Schadelin S, Kappos L, Barde YA. Measuring and validating the levels of brain-derived neurotrophic factor in human serum. eNeuro. 2018; $5(2)$.

30 Serra-Millas M. Are the changes in the peripheral brain-derived neurotrophic factor levels due to platelet activation? World J Psychiatry. 2016;6(1):84-101.
31 Webb NJ, Bottomley MJ, Watson CJ, Brenchley PE. Vascular endothelial growth factor (VEGF) is released from platelets during blood clotting: implications for measurement of circulating VEGF levels in clinical disease. Clin Sci. 1998;94(4):395-404.

32 Najm S, Löfqvist C, Hellgren G, Engström E, Lundgren $\mathrm{P}$, Hård AL, et al. Effects of a lipid emulsion containing fish oil on polyunsaturated fatty acid profiles, growth and morbidities in extremely premature infants: a randomized controlled trial. Clin Nutr ESPEN. 2017;20:17-23.

33 International Committee for the Classification of Retinopathy of Prematurity. The international classification of retinopathy of prematurity revisited. Arch Ophthalmol. 2005; 123(7):991-9.

34 Early Treatment for Retinopathy of Prematurity Cooperative Group. Revised indications for the treatment of retinopathy of prematurity: results of the early treatment for retinopathy of prematurity randomized trial. Arch Ophthalmol. 2003;121(12):1684-94.

35 Lofquist CA, Najm S, Hellgren G, Engstrom E, Savman K, Nilsson AK, et al. Association of retinopathy of prematurity with low levels of arachidonic acid: a secondary analysis of a randomized clinical trial. JAMA Ophthalmol. 2018;136:271-7.

36 Cakir B, Liegl R, Hellgren G, Lundgren P, Sun $\mathrm{Y}$, Klevebro S, et al. Thrombocytopenia is associated with severe retinopathy of prematurity. JCI Insight. 2018;3(19):e99448.

37 Sancak S, Toptan HH, Gokmen Yildirim T, Karatekin G, Ovali F. Thrombocytopenia as a risk factor for retinopathy of prematurity. Retina. 2019;39(4):706-11.

38 Kitchens CS, Weiss L. Ultrastructural changes of endothelium associated with thrombocytopenia. Blood. 1975;46(4):567-78.

39 Gore I, Takada M, Austin J. Ultrastructural basis of experimental thrombocytopenic purpura. Arch Pathol. 1970;90(3):197-205.

40 Gimbrone MA Jr, Aster RH, Cotran RS, Corkery J, Jandl JH, Folkman J. Preservation of vascular integrity in organs perfused in vitro with a platelet-rich medium. Nature. 1969; 222(5188):33-6.
41 Margraf A, Nussbaum C, Sperandio M. Ontogeny of platelet function. Blood Adv. 2019; 3(4):692-703.

42 Hartmann JS, Thompson H, Wang H, Kanekar S, Huang W, Budd SJ, et al. Expression of vascular endothelial growth factor and pigment epithelial-derived factor in a rat model of retinopathy of prematurity. Mol Vis. 2011; 17:1577-87.

43 Zhu D, Chen C, Shi W. Variations of vascular endothelial growth factor and pigment epithelial-derived factor are related to retinopathy of prematurity in human babies. West Indian Med J. 2015;65(2):251-5.

44 Fujimura H, Altar CA, Chen R, Nakamura T, Nakahashi T, Kambayashi J, et al. Brain-derived neurotrophic factor is stored in human platelets and released by agonist stimulation. Thromb Haemost. 2002;87(4):728-34.

45 Rosenfeld RD, Zeni L, Haniu M, Talvenheimo J, Radka SF, Bennett L, et al. Purification and identification of brain-derived neurotrophic factor from human serum. Protein Expr Purif. 1995;6(4):465-71.

46 Juarez CP, Muino JC, Guglielmone H, Sambuelli R, Echenique JR, Hernandez M, et al. Experimental retinopathy of prematurity: angiostatic inhibition by nimodipine, ginkgobiloba, and dipyridamole, and response to different growth factors. Eur J Ophthalmol. 2000;10:51-9.

47 Wilkinson-Berka JL, Babic S, De Gooyer T, Stitt AW, Jaworski K, Ong LG, et al. Inhibition of platelet-derived growth factor promotes pericyte loss and angiogenesis in ischemic retinopathy. Am J Pathol. 2004;164(4): 1263-73.

48 Kandasamy Y, Hartley L, Rudd D, Smith R. The association between systemic vascular endothelial growth factor and retinopathy of prematurity in premature infants: a systematic review. Br J Ophthalmol. 2017;101(1): 21-4.

49 Hellgren G, Löfqvist C, Hård AL, HansenPupp I, Gram M, Ley D, et al. Serum concentrations of vascular endothelial growth factor in relation to retinopathy of prematurity. Pediatr Res. 2016;79(1-1):70-5.

50 Battinelli EM, Markens BA, Italiano JE Jr. Release of angiogenesis regulatory proteins from platelet alpha granules: modulation of physiologic and pathologic angiogenesis. Blood. 2011;118(5):1359-69. 